\title{
Evaluation of a Novel Artificial Tear in the Prevention and Treatment of Dry Eye in an Animal Model
}

\author{
Yujing She, Jinyang Li, ${ }^{1}$ Bing Xiao, ${ }^{1}$ Huihui Lu, ${ }^{1}$ Haixia Liu, ${ }^{2}$ Peter A. Simmons, ${ }^{2}$ \\ Joseph G. Vehige, ${ }^{2}$ and Wei Chen ${ }^{1}$
}

\begin{abstract}
Purpose: To evaluate effects of a novel multi-ingredient artificial tear formulation containing carboxymethylcellulose (CMC) and hyaluronic acid (HA) in a murine dry eye model.

Methods: Dry eye was induced in mice (C57BL/6) using an intelligently controlled environmental system (ICES). CMC+HA (Optive Fusion ${ }^{\mathrm{TM}}$ ), CMC-only (Refresh Tears ${ }^{\circledR}$ ), and HA-only $\left(\right.$ Hycosan $^{\circledR}$ ) artificial tears and control phosphate-buffered saline (PBS) were administered 4 times daily and compared with no treatment ( $n=64$ eyes per group). During regimen 1 (prevention regimen), mice were administered artificial tears or PBS for 14 days (starting day 0) while they were exposed to ICES, and assessed on days 0 and 14. During regimen 2 (treatment regimen), mice exposed to ICES for 14 days with no intervention were administered artificial tears or PBS for 14 days (starting day 14) while continuing exposure to ICES, and assessed on days 0, 14, and 28. Corneal fluorescein staining and conjunctival goblet cell density were measured.

Results: Artificial tear-treated mice had significantly better outcomes than control groups on corneal staining and goblet cell density $(P<0.01)$. Mice administered $\mathrm{CMC}+\mathrm{HA}$ also showed significantly lower corneal fluorescein staining and higher goblet cell density, compared with CMC $(P<0.01)$ and HA $(P<0.05)$ in both regimens 1 and 2. Conclusions: The artificial tear formulation containing CMC and HA was effective in preventing and treating environmentally induced dry eye. Improvements observed for corneal fluorescein staining and conjunctival goblet cell retention suggest that this combination may be a viable treatment option for dry eye disease.
\end{abstract}

\section{Introduction}

$\mathbf{D}^{\mathrm{n}}$ RY EYE IS a prevalent multifactorial disease that can result from immune dysfunction, nervous system disorders, and environmental factors such as wind and low humidity, and is characterized by desiccation and ocular inflammation that alter the corneal epithelial barrier and damage the ocular surface. ${ }^{1-3}$ A hallmark of dry eye is the progressive loss of goblet cells in the nonkeratinized, stratified conjunctival epithelium. ${ }^{4}$ Goblet cell loss is related to chronic ocular inflammation and cell hyperosmolarity, which leads to further imbalance and tear film instability. ${ }^{3}$

Ocular lubricants (artificial tears) are typically used as primary treatment options for mild to moderate dry eye and in combination with other therapies for moderate to severe disease. ${ }^{5}$ Carboxymethylcellulose (CMC) sodium, a watersoluble polymer, is a well-established active ingredient in artificial tears, with efficacy in the treatment of dry eye. .,7 $^{-1}$ Hyaluronic acid (HA) is a natural component of the tear film, and similar to other soluble polymers used in artificial tears, it increases viscosity and hydrates and lubricates the ocular surface. In vitro studies have shown potential synergistic effects of combining CMC and HA in a single formulation, ${ }^{8}$ which may enhance viscosity and stabilization of the tear film. A widely available artificial tear preparation, combining CMC and HA, has not previously been available.

A variety of animal models have been developed to explore the different mechanisms underlying dry eye, as well as treatment options. ${ }^{9}$ Mouse models are most commonly used owing to the availability of different transgenic/knockout strains and panoply of antibodies. However, most models cause other complications not related to tear film abnormality, thus complicating the understanding of dry eye disease. ${ }^{9}$ The intelligently controlled environmental system (ICES)-induced murine model of dry eye has been developed, which simultaneously maintains a constant temperature, humidity, and airflow using high-capacity molecular sieves. ${ }^{10}$ The ICES model provides a greater working area than its predecessor, the controlled

\footnotetext{
${ }^{1}$ School of Ophthalmology and Optometry, Wenzhou Medical University, Wenzhou, Zhejiang, China.

${ }^{2}$ Allergan Clinical Research, Allergan, Inc., Irvine, California.
}

(C) She et al., 2015; Published by Mary Ann Liebert, Inc. This Open Access article is distributed under the terms of the Creative Commons Attribution Noncommercial License (http://creativecommons.org/licenses/by-nc/4.0/) which permits any noncommercial use, distribution, and reproduction in any medium, provided the original author(s) and the source are credited. 
environment chamber model, ${ }^{11}$ thus increasing consistency by allowing evaluation of larger sample sizes. The model has been valuable in assessing novel agents for treatment of dry eye. ${ }^{12-15}$

Using the ICES-induced murine dry eye model, we compared the effects of a new multi-ingredient artificial tear formulation, containing $0.5 \% \mathrm{CMC}$ and $0.1 \% \mathrm{HA}$, with standard artificial tear formulations, containing either $0.5 \%$ $\mathrm{CMC}$ or $0.1 \% \mathrm{HA}$ alone, on corneal fluorescein staining and conjunctival goblet cell density.

\section{Methods}

\section{ICES-induced murine dry eye model}

Female C57BL/6 mice (aged 4-6 weeks) were carefully selected from the Animal Breeding Unit at Wenzhou Medical College, Wenzhou, Zhejiang, China, for inclusion in the study using slit-lamp microscopy and corneal fluorescein staining. Only healthy mice with no corneal infections, infiltration, or leukoma, and with total corneal fluorescein staining scores $<10$ were used for the study. All animal procedures were approved by the Animal Care and Ethics Committee of Wenzhou Medical College, Wenzhou, Zhejiang, China, and adhered to the Association for Research in Vision and Ophthalmology Statement for the Use of Animals in Ophthalmic and Vision Research.

Dry eye was induced in the selected mice using the previously established process described as the ICES-induced murine dry eye model. ${ }^{10}$ In this model, animals were placed in a chamber that maintained a relative humidity of $15.3 \% \pm 3.0 \%$, an air flow of $2.1 \pm 0.2 \mathrm{~m} / \mathrm{s}$, and a temperature of $21^{\circ} \mathrm{C}-23^{\circ} \mathrm{C}$. These environmental parameters were monitored daily for accuracy. Mice are known to develop clinical signs of dry eye, similar to those found in humans with dry eye disease, when exposed to the low humidity maintained by the ICES chamber. ${ }^{10,11}$

\section{Treatments and administration schedules}

Three artificial tear formulations, $0.5 \% \mathrm{CMC}+0.1 \% \mathrm{HA}$ (Optive Fusion $^{\mathrm{TM}}$, Purite ${ }^{\circledR}$ preserved; Allergan, Inc., Irvine, CA), $0.5 \%$ CMC (Refresh Tears ${ }^{\circledR}$, Purite preserved; Allergan, Inc.), $0.1 \%$ HA $\left(\right.$ Hycosan $^{\circledR}$, preservative free; Ursapharm, Saarbrücken, Germany), and a control solution, phosphatebuffered saline (PBS), were administered topically 4 times daily in each eye. Each formulation was administered through 2 regimens while the mice were housed in the ICES chamber. During regimen 1 (prevention regimen), eye drops were administered for 14 days starting from day 0 . During regimen 2 (treatment regimen), after the mice had been housed in the ICES chamber for 14 days with no intervention, eye drops were administered for 14 days starting from day 14 while the mice continued to be housed in the ICES chamber. Animals housed in the ICES chamber with no treatment (NT) served as positive controls, and healthy mice housed in a normal environment and receiving NT (normal) served as normal controls. At day 0 , during both regimens 1 and 2, each treatment (CMC+HA, CMC, HA, PBS) and control (NT, normal) group consisted of a total of 64 eyes $(n=32$ mice).

\section{Corneal fluorescein staining}

Corneal fluorescein staining was assessed in all eyes on days 0,14 , and 28 after instilling $0.5 \mu \mathrm{L}$ of $5 \%$ fluorescein
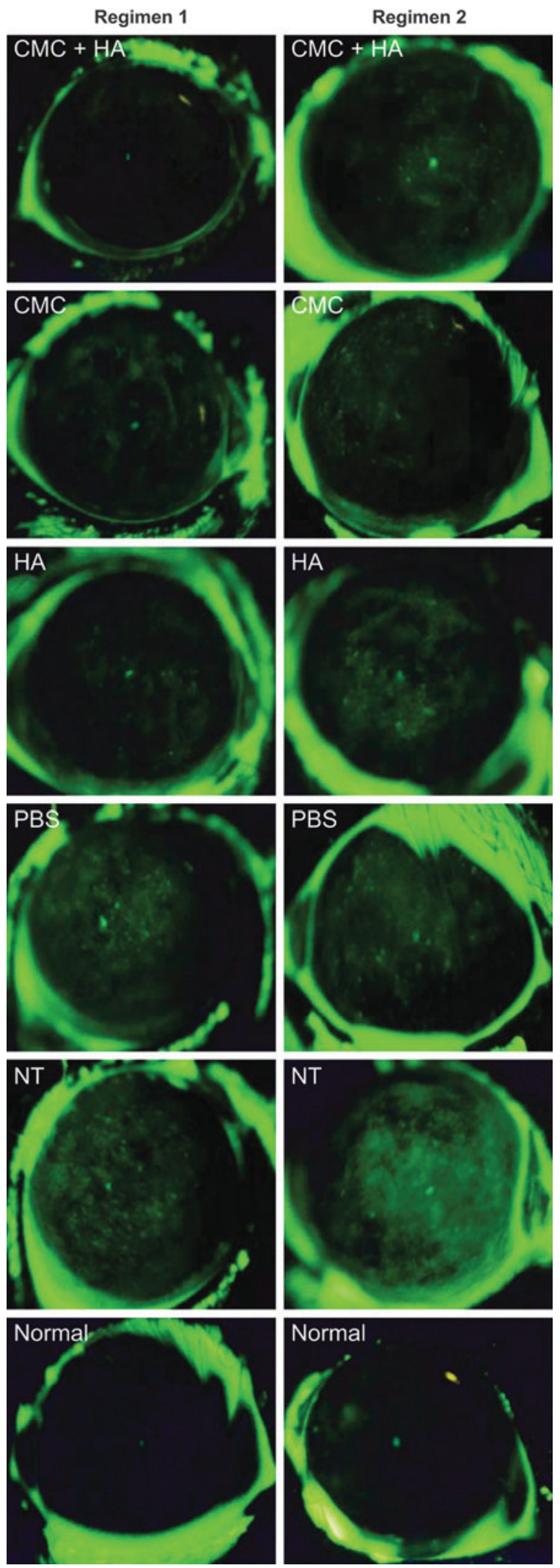

FIG. 1. Representative corneal fluorescein staining in mice assessed on day 14 (regimen 1) and day 28 (regimen 2) after placement in the ICES dry eye chamber and treatment with topical artificial tear formulations. CMC, carboxymethylcellulose; HA, hyaluronic acid; ICES, intelligently controlled environmental system; PBS, phosphate-buffered saline; NT, no treatment. 
solution ( $25 \mathrm{mg}$ fluorescein sodium in $0.5 \mathrm{~mL}$ PBS) into the inferior conjunctival sac using a micropipette. The cornea was examined using slit-lamp microscopy with cobalt blue light immediately following fluorescein instillation. The stained area was assessed and graded using the 2007 Dry Eye Work Shop-recommended grading system ${ }^{16}$; the corneal surface was divided into 5 regions and staining was graded on a scale of $0-4$ ( 0 dots, grade $0 ; 1-5$ dots, grade $1 ; 6-15$ dots, grade 2; 16-30 dots, grade 3; and $>30$ dots, grade 4). The total score from the 5 regions was recorded for analysis.

\section{Periodic acid-Schiff staining for goblet cells}

Mice from each treatment group were sacrificed using an overdose mixture of ketamine and xylazine for histological evaluation on day 14 (during regimen 1) and day 28 (during regimen 2) after the start of ICES-induced dry eye. Mice in the treatment $(\mathrm{CMC}+\mathrm{HA}, \mathrm{CMC}, \mathrm{HA}, \mathrm{PBS})$ and control (NT, normal) groups were euthanized at the same time for comparison. Twelve enucleated eyes from sacrificed mice $(n=6)$ in each group were fixed in $10 \%$ formalin, dehydrated, and embedded in paraffin. To quantify goblet cells in the conjunctiva, $4-\mu \mathrm{m}$ cross sections were stained with the periodic acid-Schiff reagent. The number of goblet cells was assessed under a microscope (Axio Imager Z1; Carl Zeiss Meditec, Oberkochen, Germany).

\section{Statistical analyses}

The Mann-Whitney $U$-test was used to compare the differences in fluorescein staining scores and analysis of variance was used to compare goblet cell density between the treatment and control groups; $P<0.05$ was considered statistically significant. Analyses were performed using SPSS 18.0 software (IBM, Armonk, NY).

\section{Results}

\section{Corneal fluorescein staining}

Figure 1 shows representative corneal fluorescein staining in mice housed in the ICES chamber after receiving treatment with artificial tears. All artificial tear formulations $(\mathrm{CMC}+$ $\mathrm{HA}, \mathrm{CMC}$, and HA) improved corneal staining compared with the control groups (PBS and NT) at day 14 during regimen 1 and day 28 (14 days after treatment initiation) during regimen 2. Quantitatively, there were no differences in corneal staining scores observed at the beginning of the ICESinduced dry eye process (day 0) during both regimens 1 and 2, and on day 14 when treatment with artificial tears was initiated during regimen 2 (Fig. 2a, b). After 14 days of treatment with artificial tears in both regimens 1 and 2, fluorescein corneal staining was significantly reduced in mice receiving $\mathrm{CMC}+\mathrm{HA}, \mathrm{CMC}$ only, or HA only compared with mice in the PBS-treated and NT control groups $(P<0.01)$. The greatest reduction in corneal staining was observed in mice treated with artificial tears containing $\mathrm{CMC}+\mathrm{HA}$ followed by $\mathrm{HA}$ only during both test regimens. The artificial tear formulation containing $\mathrm{CMC}+\mathrm{HA}$ significantly reduced corneal staining compared with formulations containing CMC only $(P<0.01)$ and HA only $(P<0.05)$ at day 14 (regimen 1; Fig. 2a) and day 28 (regimen 2; Fig. 2b). During regimen 2 (treatment regimen), the artificial tear formulation containing HA only significantly decreased corneal staining compared with the formulation containing CMC only $(P<0.05$; Fig. $2 b)$.

\section{Conjunctival goblet cell quantification}

In the normal group of mice (not housed in the ICES chamber), the mean ( \pm standard deviation) number of goblet cells was $193.5 \pm 11$. Mice housed in the ICES chamber for 28 days (regimen 2) demonstrated more dramatic decreases in conjunctival goblet cells than mice housed for 14 days (regimen 1; Fig. 3). Quantitatively, the number of goblet cells was greater in ICES-housed mice following treatment with artificial tear formulations containing $\mathrm{CMC}+\mathrm{HA}, \mathrm{CMC}$ only, or HA only compared with mice receiving PBS or NT (Fig. 4). Treatment with the artificial tear formulation containing $\mathrm{CMC}+\mathrm{HA}$ resulted in significantly higher numbers of goblet cells at day 14 (regimen 1; Fig. 4a) and day 28 (regimen 2; Fig. 4b) compared with formulations containing CMC only $(P<0.01)$ or HA only $(P<0.05)$.
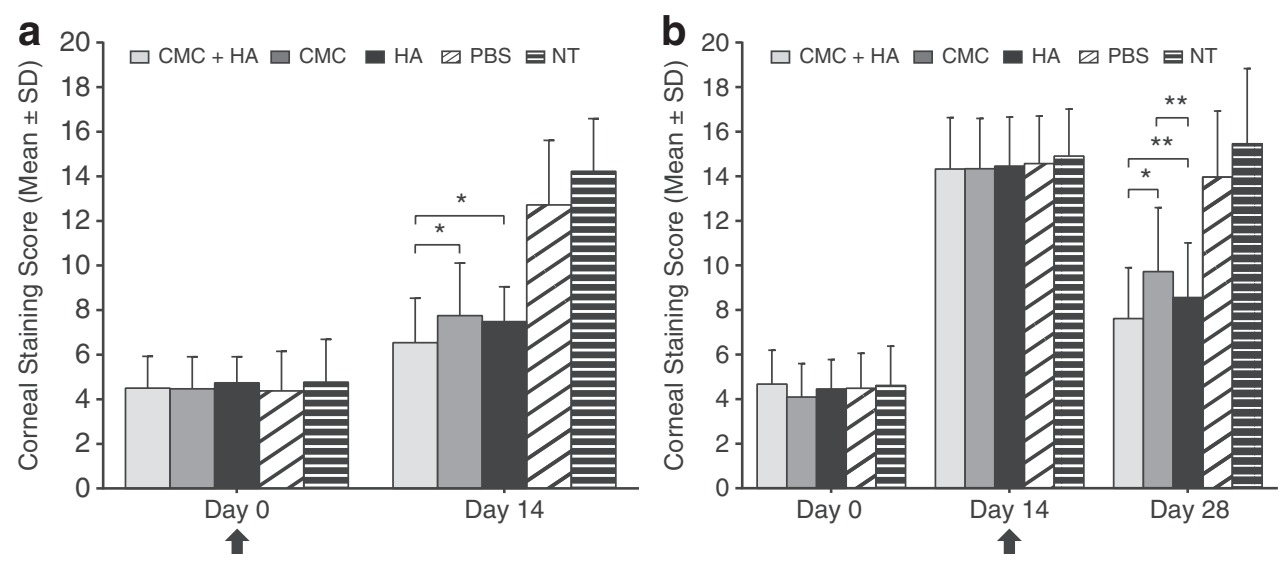

FIG. 2. Mean corneal fluorescein staining in mice (64 eyes, $n=32$ ) housed in the ICES chamber. (a) Regimen 1: artificial tear formulations were administered to mice immediately after placement in the ICES dry eye chamber, and corneal staining was evaluated at days 0 and 14. (b) Regimen 2: artificial tear formulations were administered to mice after 14 days of initiating ICES-induced dry eye, and corneal staining was evaluated at days 0,14 , and 28 after continued exposure to the ICES chamber. Arrows indicate when administration of artificial tears was initiated for mice treated in regimen 1 and regimen 2 . $* P<0.01$; $* * P<0.05$ between treatment groups compared on the same day. 


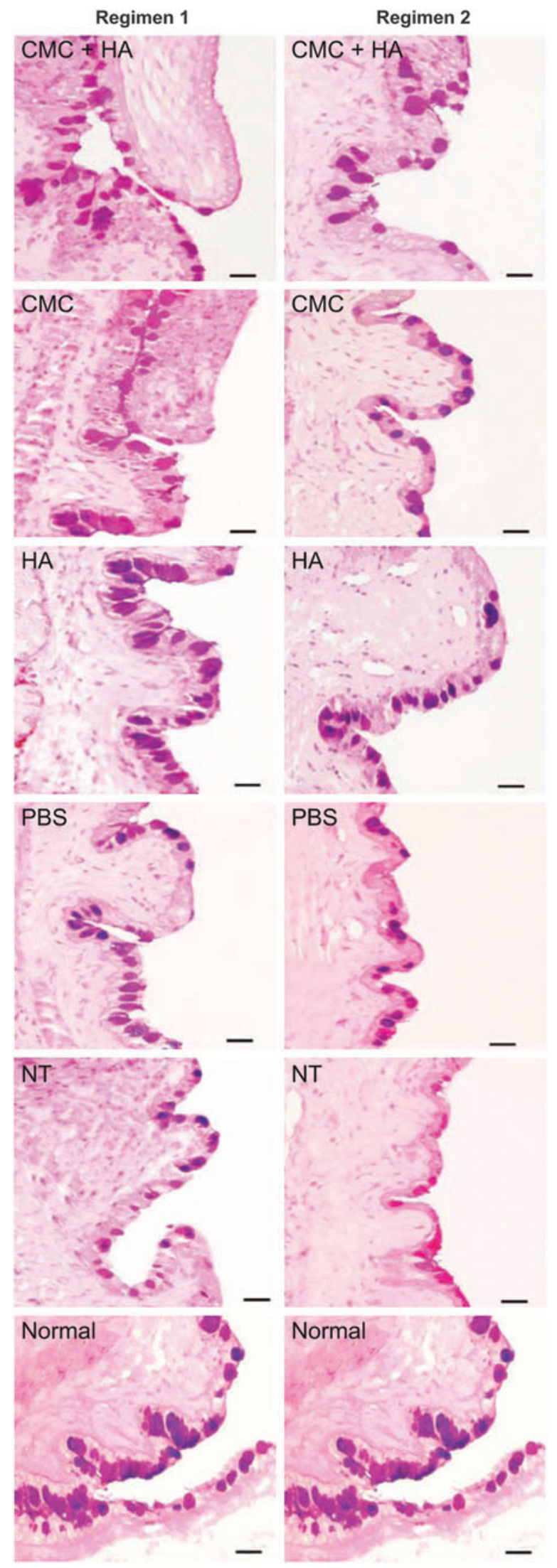

FIG. 3. Representative periodic acid-Schiff staining of conjunctival goblet cells on day 14 (regimen 1) and day 28 (regimen 2) of exposure to the ICES dry eye chamber and treatment with topical artificial tear formulations. Magnification, 40×; scale bars, $20 \mu \mathrm{m}$.

\section{Discussion}

The ICES-induced murine model of dry eye employed in this study has been proposed as a model for mild to moderate human dry eye disease, as it relies solely on environmental modification without pharmacological intervention. ${ }^{10,12-14}$ Animals housed in the ICES chamber had increased corneal staining and decreased conjunctival goblet cell density after 14 days of exposure to the low humidity environment. Mice receiving treatment with artificial tear preparations $(\mathrm{CMC}+$ $\mathrm{HA}, \mathrm{CMC}$, or $\mathrm{HA}$ ) for 14 days, either during the prevention regimen or the treatment regimen in the low humidity environment maintained by the ICES chamber, demonstrated a significant reduction in corneal staining and retention of goblet cell density compared with control animals. Instillation of the novel artificial tear formulation combining CMC and HA resulted in the most significant reduction in development of dry eye than other formulations tested; less fluorescein staining and greater numbers of conjunctival goblet cells were observed in eyes treated with the formulation containing CMC and HA compared with formulations containing CMC only and HA only.

An important function of artificial tear preparations is to reduce potential corneal damage by supplementing the tear film and lubricating the ocular surface. ${ }^{17-19}$ Both CMC and HA are well-established components of artificial tears. CMC has been shown to bind to ocular surface cells and accelerate wound healing in model systems. ${ }^{6,7,20,21}$ The glycosaminoglycan HA represents a distinct class of polymeric agents owing to its intrinsic properties of water retention, viscoelasticity, and promotion of corneal epithelial wound healing. ${ }^{22}$ Similar to CMC, HA also has been reported to improve corneal and conjunctival staining in patients with dry eye. ${ }^{23-25}$ In current artificial tear formulations, the viscosity has been varied to influence ocular surface retention time and enhance cellular protection. ${ }^{26}$

Potential synergistic effects of combining CMC and HA in a single formulation have been demonstrated in vitro. ${ }^{8}$ Synergistic effects between the polymers enhance the shear thinning property of HA; under low-shear conditions characteristic of the tear film between blinks, CMC and HA polymer entanglement increases viscosity. Higher viscosity promotes stabilization of the tear film and retention of the eye drop in the eye, which optimizes ocular hydration. Conversely, the polymer combination produces reduced viscosity in highshear conditions, such as during blinking, potentially improving ocular comfort by reducing stickiness and blurring. Improved viscosity and ocular hydration resulting from interactions between CMC and HA polymers likely contributed to the reduced development of dry eye signs with CMC plus HA observed in the present study. The combination of polymers present in the artificial tear with CMC and HA was more effective despite the fact that the formulation contains a preservative, while the formulation with HA only had no preservative. This suggests that either the dissipating preservative present in the artificial tear formulation containing CMC and HA had minimal impact in the ICES-induced dry eye animal model or the benefit of the polymer combination substantially outweighed any deleterious effects caused by the preservative.

Although both artificial tear formulations with CMC only and HA only significantly reduced corneal staining compared to PBS or NT during both the prevention and treatment regimens, we also observed a significantly better effect with 

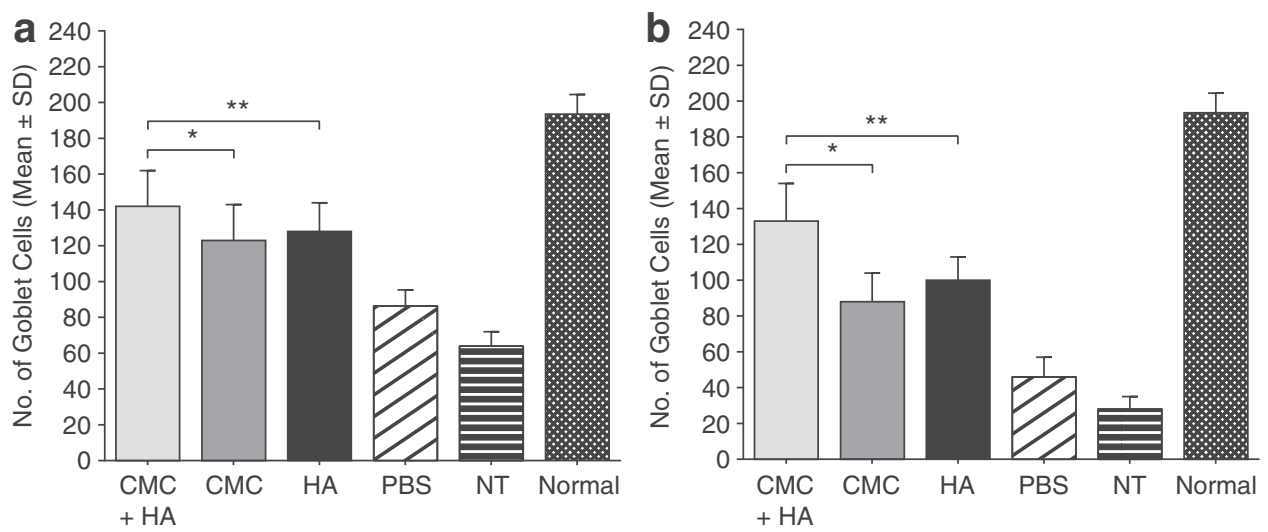

FIG. 4. Mean number of conjunctival goblet cells in mice (12 eyes, $n=6)$ housed in the ICES chamber. (a) Regimen 1: artificial tear formulations were administered to mice immediately after placement in the ICES dry eye chamber and goblet cells were quantified at day 14. (b) Regimen 2: artificial tear formulations were administered to mice after 14 days of exposure to the ICES dry eye chamber and goblet cells were quantified at day 28 after continued exposure to the ICES chamber. $* P<0.01 ; * * P<0.05$ between treatment groups compared on the same day.

HA alone than CMC alone during both the prevention and treatment regimens. The clinical performance of artificial tear formulations with CMC only and HA only has been compared in preservative-free ${ }^{19}$ and preserved ${ }^{25}$ preparations in prospective, randomized clinical trials, and both studies showed similar results between the 2 polymers. However, in our study, the formulation with HA only was preservative free, whereas the formulation with CMC only was preserved, which may partially explain the better results observed with HA alone.

The novel artificial tear combining CMC and HA demonstrated a benefit in ICES-induced dry eye and may be an important addition to treatment options available for patients with dry eye disease. The current study was conducted using a murine model that relies on controlling environmental factors to induce dry eye. Although animal models have inherent limitations, the results of the present study are consistent with clinical investigation of artificial tear formulations containing $\mathrm{CMC}$ and HA or CMC only for treatment of dry eye. ${ }^{27}$

\section{Conclusion}

Using a murine model of environmentally induced dry eye, a recently developed artificial tear preparation containing both $\mathrm{CMC}$ and $\mathrm{HA}$ effectively improved clinical signs of dry eye; the combination was superior to products containing either $\mathrm{CMC}$ or $\mathrm{HA}$ alone in reducing corneal staining and loss of conjunctival goblet cells.

\section{Acknowledgments}

This study was sponsored by Allergan, Inc., Irvine, CA. Writing and editorial assistance was provided to the authors by Kakuri Omari, PhD, of Evidence Scientific Solutions, Philadelphia, PA, and funded by Allergan, Inc. All authors met the International Committee of Medical Journal Editors authorship criteria. Neither honoraria nor payments were made for authorship.

\section{Author Disclosure Statement}

W.C., Y.S., J.L. B.X., and H.L. have no competing conflicts of interest; H.L., P.A.S., and J.G.V. are employees of Allergan, Inc., Irvine, CA.

\section{References}

1. The definition and classification of dry eye disease: report of the Definition and Classification Subcommittee of the International Dry Eye WorkShop. Ocul. Surf. 5:75-92, 2007.

2. The epidemiology of dry eye disease: report of the Epidemiology Subcommittee of the International Dry Eye WorkShop. Ocul. Surf. 5:93-107, 2007.

3. Song, X.J., Li, D.Q., Farley, W., et al. Neurturin-deficient mice develop dry eye and keratoconjunctivitis sicca. Invest. Ophthalmol. Vis. Sci. 44:4223-4229, 2003.

4. Baudouin, C., Haouat, N., Brignole, F., Bayle, J., and Gastaud, P. Immunopathological findings in conjunctival cells using immunofluorescence staining of impression cytology specimens. Br. J. Ophthalmol. 76:545-549, 1992.

5. Behrens, A., Doyle, J.J., Stern, L., et al. Dysfunctional Tear Syndrome Study Group. Dysfunctional tear syndrome: a Delphi approach to treatment recommendations. Cornea. 25:900-907, 2006.

6. Grene, R.B., Lankston, P., Mordaunt, J., et al. Unpreserved carboxymethylcellulose artificial tears evaluated in patients with keratoconjunctivitis sicca. Cornea. 11:294-301, 1992.

7. Simmons, P.A., and Vehige, J.G. Clinical performance of a mid-viscosity artificial tear for dry eye treatment. Cornea. 26:294-302, 2007.

8. Simmons, P.A., Beard, B.J., and Vehige, J.G. Optimizing viscosity of ophthalmic solutions with the combination of two polymers. Presented at the 7th International Conference on the Tear Film \& Ocular Surface: Basic Science and Clinical Relevance; September 18-21, 2013; Taormina, Italy.

9. Schrader, S., Mircheff, A.K., and Geerling, G. Animal models of dry eye. Dev. Ophthalmol. 41:298-312, 2008.

10. Chen, W., Zhang, X., Zhang, J., et al. A murine model of dry eye induced by an intelligently controlled environmental system. Invest. Ophthalmol. Vis. Sci. 49:1386-1391, 2008.

11. Barabino, S., Shen, L., Chen, L., et al. The controlledenvironment chamber: a new mouse model of dry eye. Invest. Ophthalmol. Vis. Sci. 46:2766-2771, 2005.

12. Chen, W., Zhang, X., Liu, M., et al. Trehalose protects against ocular surface disorders in experimental murine dry eye through suppression of apoptosis. Exp. Eye Res. 89: 311-318, 2009. 
13. Li, J., Roubeix, C., Wang, Y., et al. Therapeutic efficacy of trehalose eye drops for treatment of murine dry eye induced by an intelligently controlled environmental system. Mol. Vis. 18:317-329, 2012.

14. Chen, W., Zhang, X., Li, J., et al. Efficacy of osmoprotectants on prevention and treatment of murine dry eye. Invest. Ophthalmol. Vis. Sci. 54:6287-6897, 2013.

15. Zheng, Q., Ren, Y., Reinach, P.S., et al. Reactive oxygen species activated NLRP3 inflammasomes prime environmentinduced murine dry eye. Exp. Eye Res. 125:1-8, 2014.

16. Baudouin, C. [A new approach for better comprehension of diseases of the ocular surface]. J. Fr. Ophtalmol. 30:239246, 2007.

17. Aragona, P., Papa, V., Micali, A., Santocono, M., and Milazzo, G. Long term treatment with sodium hyaluronatecontaining artificial tears reduces ocular surface damage in patients with dry eye. Br. J. Ophthalmol. 86:181-184, 2002.

18. Doughty, M.J., and Glavin, S. Efficacy of different dry eye treatments with artificial tears or ocular lubricants: a systematic review. Ophthalmic Physiol. Opt. 29:573-583, 2009.

19. Lee, J.H., Ahn, H.S., Kim, E.K., and Kim, T.-I. Efficacy of sodium hyaluronate and carboxymethylcellulose in treating mild to moderate dry eye disease. Cornea. 30:175-179, 2011.

20. Garrett, Q., Simmons, P.A., Xu, S., et al. Carboxymethylcellulose binds to human corneal epithelial cells and is a modulator of corneal epithelial wound healing. Invest. Ophthalmol. Vis. Sci. 48:1559-1567, 2007.

21. Garrett, Q., Xu, S., Simmons, P.A., et al. Carboxymethyl cellulose stimulates rabbit corneal epithelial wound healing. Curr. Eye Res. 33:567-573, 2008.

22. Gomes, J.A., Amankwah, R., Powell-Richards, A., and Dua, H.S. Sodium hyaluronate (hyaluronic acid) promotes migration of human corneal epithelial cells in vitro. $\mathrm{Br} . \mathrm{J}$. Ophthalmol. 88:821-825, 2004.

23. Vogel, R., Crockett, R.S., Oden, N., Laliberte, T.W., Molina, L.; Sodium Hyaluronate Ophthalmic Solution Study
Group. Demonstration of efficacy in the treatment of dry eye disease with $0.18 \%$ sodium hyaluronate ophthalmic solution (Vismed, Rejena). Am. J. Ophthalmol. 149:594$601,2010$.

24. Baeyens, V., Bron, A., Baudouin, C.; Vismed/Hylovis Study Group. Efficacy of $0.18 \%$ hypotonic sodium hyaluronate ophthalmic solution in the treatment of signs and symptoms of dry eye disease. J. Fr. Ophtalmol. 35:412419, 2012.

25. Baudouin, C., Cochener, B., Pisella, P.J., et al. Randomized, phase III study comparing osmoprotective carboxymethylcellulose with sodium hyaluronate in dry eye disease. Eur. J. Ophthalmol. 22:751-761, 2012.

26. Paugh, J.R., Nguyen, A.L., Ketelson, H.A., Christensen, M.T., and Meadows, D.L. Precorneal residence time of artificial tears measured in dry eye subjects. Optom. Vis. Sci. 85:725-731, 2008.

27. Simmons, P.A., Liu, H., Carlisle-Wilcox, C., Shi, G., and Vehige, J.G. A multi-center, double-masked, randomized study to compare the safety, efficacy, and acceptability of two investigational eye drop formulations to cellufluid for three months in subjects with dry eye disease. Presented at the 4th EuCornea Congress; October 4-5, 2013; Amsterdam RAI, the Netherlands.

Received: March 25, 2015

Accepted: July 3, 2015

Address correspondence to: Dr. Wei Chen School of Ophthalmology and Optometry Wenzhou Medical University 270 Xueyuan West Road Wenzhou 325027 Zhejiang China

E-mail: chenweiwmc@gmail.com 